\title{
Learning Aids of Risk Assessment Apps for Practical Engineering Student: A Case Study
}

\author{
https://doi.org/10.3991/ijet.v14i24.11981 \\ Nor Kamaliana Khamis ${ }^{\left({ }^{\varpi}\right)}$, Amirul Mukhlish Abdul Azam \\ Universiti Kebangsaan Malaysia, Selangor, Malaysia \\ kamaliana@ukm.edu.my
}

\begin{abstract}
In today's learning approaches for engineering students, practical courses frequently complement traditional theoretical lectures. This practical element allows an early perspective into the real world of engineering, augments student motivation, and enables students to acquire soft skills early. This paper aims to demonstrate the usage of one of the ergonomics risk assessment (ERA) apps that has been learned in the class; which can also be used as an assessment aid in student's practical sessions in the workplace. This apps has been evaluated for its effectiveness by a group of students in the classroom setting prior to be used in the actual workplace. Evaluation results show that this learning aid improves student's communication skills and assists companies to improve their working conditions. Based on the effectiveness assessment, students find that this apps is really useful for their assessment as it provides fast calculation on the results and can store it in the drive. Based on the practical session in the workplace, the findings from the ERA apps show that the current working condition in this study is under medium ergonomic risk exposure level. Therefore, there is a need to improve the working condition by considering improvement on the workplace environment as well as interaction between worker and tools for maintenance. This app is indeed beneficial for practical engineering students as a peer learning process and for industry in terms of work place improvements. In conclusion, this paper describes how this risk assessment app helps induce student to think as an actual engineer and also to solve real-world tasks with limited resources.
\end{abstract}

Keywords-Mobile apps, educational learning, practical, student

\section{$1 \quad$ Introduction}

\subsection{Adaptation of practical engineering student in the real workplace}

Practical learning is one approach that has proved to be effective for student understanding, experience, and application in real working environments. There are five advantages of practical learning which involving provide opportunity by hands on learning, culture and practices exposure, real life job experience, application of learned theory, improve the technical and soft skill, and achieve the industry expecta- 
tion [1]. It is easy for engineers to run software simulations to fix problems. However, they did not have a practical understanding to initially setup the problem. It is important for engineers to learn practically in order for them to learn how to operate their tools. The emergence of new technologies brings more effective learning and flexibility [2]. It is important to know how technology is used to support learners, make learning more efficient and experiences more memorable, and improve access to ideas and information [3]. Technology is a medium that is highly interactive, individualized, more flexible, and more accessible [4]. Exploration into the usage of technology in the form of mobile devices nowadays is significant as it is in line with the current need of the student generation. Due to flexibility and accessibility of technology particularly refers to mobile learning, student has access to learn from anywhere.

Smartphones, tablets and laptops can be used to improve teaching and learning, while helping students to acquire digital skills and competencies. Smartphones, and other mobile gadgets, can provide convenient informative resources to support learning activities that can be accessed remotely or face to face between educator and students during classes $[5,6,7,8]$. The increase in Internet usage among organisations, either for learning activities, for training or working purposes, is indeed noticeable today [9-12]. From an education perspective, it is time to change the learning education system from traditional to modern. In this case, it needs to move from manual and direct methods to non-traditional spaces using currently evolving worldwide technologies. The learning experience should be conducted in non-traditional spaces using integrated software and mobile devices or online systems shared with students or respective parties [8]. Williams \& Pence highlighted in their research that the use of mobile devices can help to ease the empirical studies and provides more autonomous learning [8]. The mobile devices certainly provides remarkable solution in education innovation to meet the requirement of the Education 4.0.

In term of education perspective, safety is an important issue in engineering lesson and work practice. Therefore, students should consider safety issue in all activities especially in their field work. Risk factor assessment is important in order to decide safety level for each activity [4]. However, manual risk factor assessment is tedious and time consuming. Thus, students as well as educators tend to ignore this important issue. For Science, Technology, Engineering and Mathematics (STEM) education, Human Factors Engineering (HFE) or Ergonomics is one of the example that highlight safety issue when performing the work task in the Engineering curriculum. Basically, HFE does have a unique place amongst the modern discipline of study and practice [2]. HFE can be describe as a study of work in term of multidisciplinary field such as engineering design, engineering management, physiology (social science), biomechanics, and kinesiology (health) [13-14]. The most popular topic in the HFE is the risk assessment. Up to this date, majority of the assessment related to HFE risk factor were performed manually. In this case, it refers to a pen and paper based measurement. Therefore, it is quite time-consuming and difficult for the user to perform the assessment with limited of time in study period.

This assessment is really useful not for learning only, however, majority of the industrial organisation use it to improve their working productivity. Ergonomic workplace is one of the important things in industry to improve the productivity in an or- 
ganization. The ergonomic risk is one of low productivity causes in working activity [15]. In the HFE risk factors assessment perspective, it is really useful to identify any problem related to working condition among the people [16]. However, till this date, common assessment tools related to HFE risk factor were performed by using pen and paper based measurement. As a result, it is really not very user friendly and timeconsuming. In addition, if there are many observations of working task in the same time, the observation task will be quite handful and the observer will take extra time to analyse the data. Consequently, all these factors might influence the learning environment in this topic, due to time consuming and lack of information in a single source. Therefore, the idea to develop user friendly and interactive assessment tools in the mobile device and apps for student and industry usage is encouraged with the recent trend towards Education 4.0.

With clear understanding and knowledge, hopefully this mobile tool can assist the researcher, academician and practitioner in many organisations to deal with the safety assessment in the future in the user-friendly setting. Furthermore, these integrated and mobile apps tools can engage the Generation $\mathrm{Z}$ characteristics that prefers any learning medium in the iconised-form. As a result, these systems can assist the user, particularly practical engineering student to deliver the contents in more presentable way and systematic to the industry.

\subsection{Effectiveness of the mobile application to replace the conventional method of learning form}

There is a broad consensus to change the learning process from conservative form to digital form. Careful evaluations should be performed to investigate the effectiveness of the new learning methods. However, up to this date, digital form or known as the apps form is widely applied particularly in education setting. The suitable use of materials and tool in providing the contents of the courses is undeniably helpful in ensuring the learning process more meaningful. Majority of past studies examined students' readiness and perceptions towards the use of mobile technology in their classroom [16-20]. Findings from the past studies showed that students provides positive feedback on the usage of mobile device such as mobile phone and tablet for learning process.

Overall, this article concentrates on the introduction and application of apps in the classroom and workplace by practical engineering students as a case study. Prior to using it outside of the university, validation of the apps with the expert and an assessment to determine effectiveness of this apps was carried out among students in the classroom setting. With regards to the preceding discussion, the following research questions (RQs) were determined in this study:

1. To what extend the mobile apps capable to improve learning process in university subject?

2. To what extend the apps capable to assist the students in their learning environment, either in the classroom setting or outside of the classroom? 
3. What are the benefits of the mobile apps for other parties (outside university) that dealt directly with the student?

\section{Methodology}

This section provides five main components on how this study was conducted. Basically, a few experts and a group of students were involved in this study to provide the comments, feedbacks and their perception on the practicality and benefits of this mobile apps. Then, selected company was visited and observed by the student to use this mobile apps as one of their actual and practical learning based on the real working condition.

\subsection{Research flow chart}

This section explains the methodology of how ERA methods have been reviewed and ends with the application's evaluation and feedback. The flowchart in Figure 1 represents the process clearly, from the starting process to the end line. Figure 1 shows the research flow chart and it will be explained in each subsection under Methodology part.

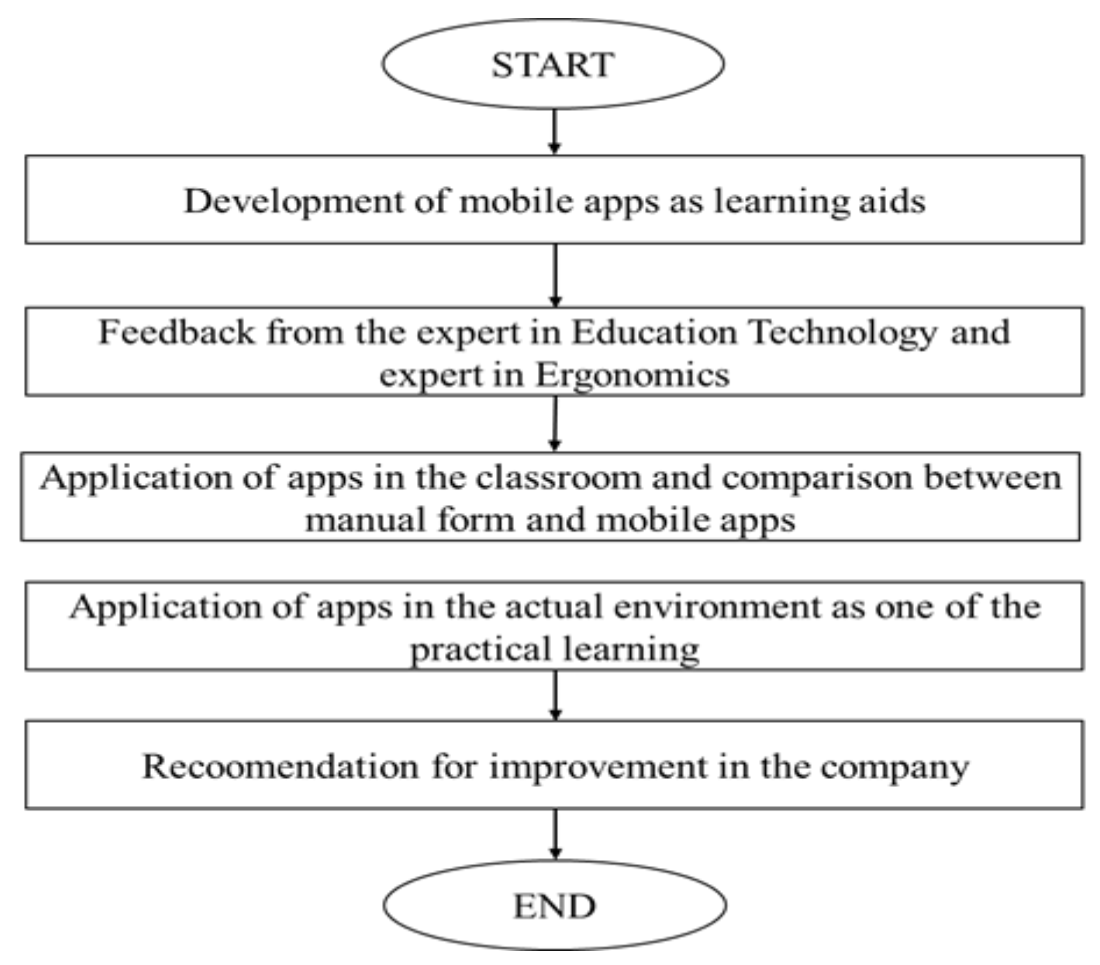

Fig. 1. Flowchart of mobile apps development and assessment 


\subsection{Development of mobile apps as learning aids}

In this study, Simple Ergonomics Risk Assessment (SERA) form was converted to digital feature in the form of apps. SERA is a risk evaluation method to evaluate human work by considering comprehensive factors [16]. SERA was selected because this assessment is among the recent and validated ERA methods that have been developed by Malaysian researchers [16]. In addition, this method covers many important factors related to working condition, consisting of posture, force (for the left and right body position), breaks, work pace and other significant factors in the workplace. Figure 2 shows some of observations that need to be performed based on the SERA manual form.

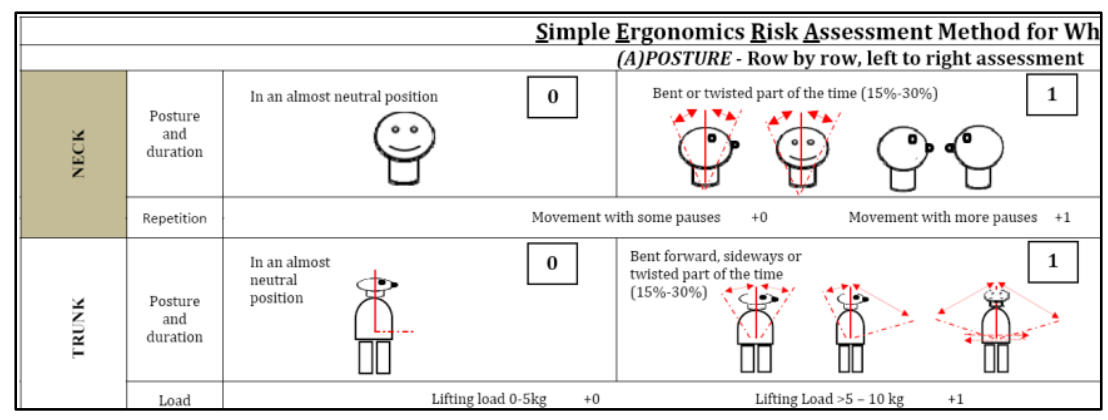

Fig. 2. Part of diagram in the SERA manual form from Sukadarin et al. [12]

The mobile apps development process was programmed by using Android platform. Figure 3 shows the SERA interfaces that are installed in the Android mobile apps. As shown in Figure 3(a), the apps starts with the front page. The user is required to click on the 'Start now' button to enter into the app. The user is then required to complete the information regarding the observed workplace and the respondent in this observation as shown in Figure 3(b). The third interface as shown in Figure 3(c) depicts the main factors of the SERA method, consisting of posture, force, breaks, work pace and other factors. There are seven body parts need to be evaluated in this assessment. Then, force level and exerted force based on time are the main factors for force. The third and fourth factor are regarding break time and work pace. The user requires to choose one answer for each factor. The last factor is related to another significant factors related to work condition. For instance, if there is insufficient humidity in the workplace, hence, one factor is presented in this observation. Basically, each factor has different scores. The SERA app can generate immediate results after the completion of the observation session, as illustrated in Figure 3(d). In addition, based on the results, it will shows the stage of working conditions by referring to exposure score. There are three levels of exposure score for SERA method: i) Low exposure: the total score is in between 1 to 22, ii) Medium exposure: the total score is in between 23 to 45, and iii) exposure: the total score is above 45 [16]. All of these results can be stored in the specified folder during installation of this application. 


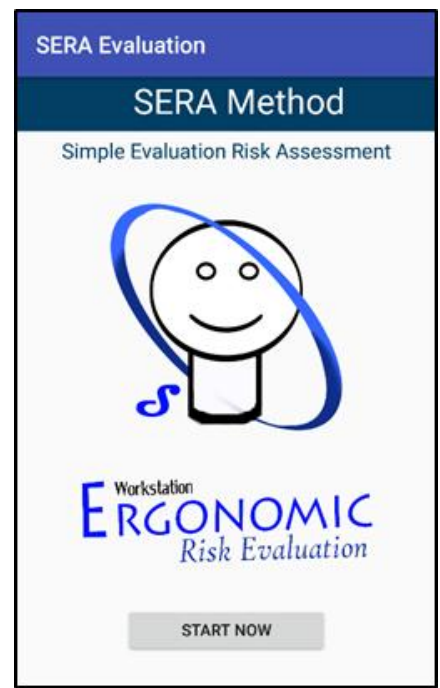

(a) Main interface

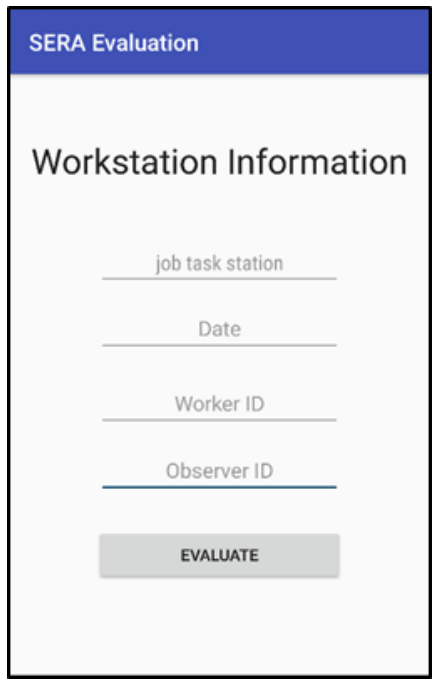

(b) Workstation information

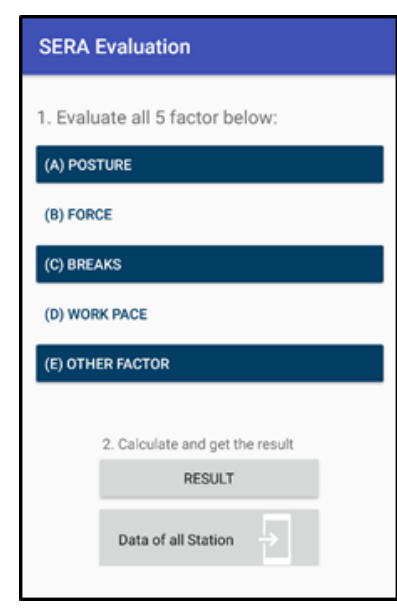

(c) Evaluation on five factors

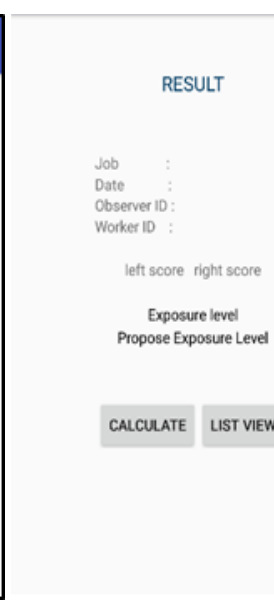

(d) Result
04 feb $2018,11 \cdot 2427 \mathrm{pm}$

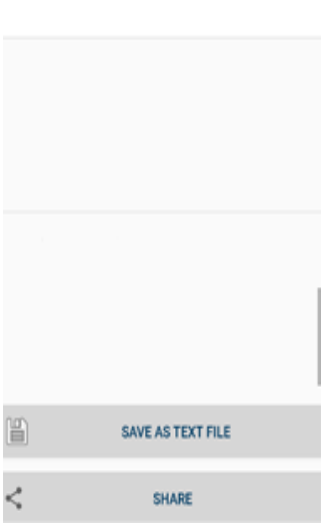

(e) Data storage

Fig. 3. SERA mobile apps interface

The data is then automatically collected in a list view, as shown in Figure 4. Next, a list view of all data is saved in a .txt file by clicking the "save as text file" button. A message stating "/Internal Storage/SERA-File/filename.txt" then appears (the file is named using the current date and time, which is then saved in the SERA-File internal storage). The user then clicks the 'share' button to open Google Drive and share the .txt file in the storage cloud (as shown in Figure 4). 


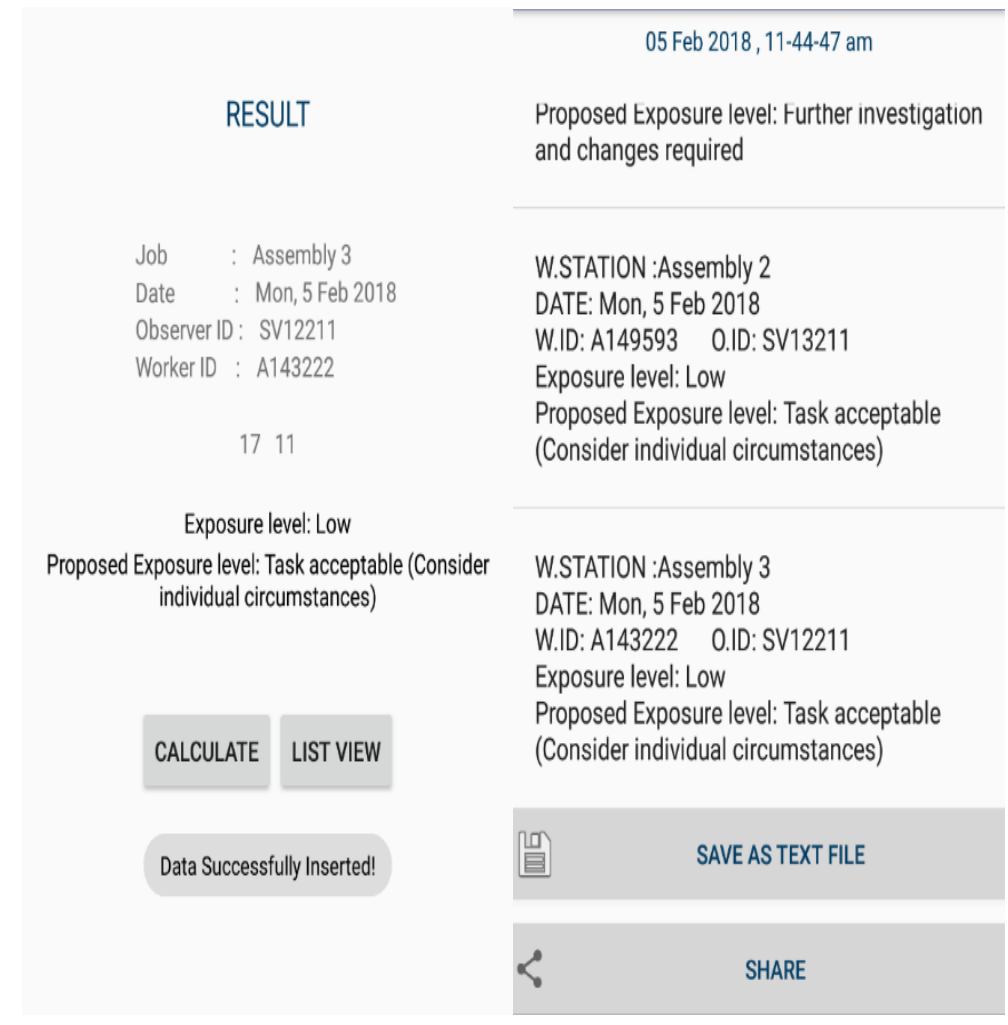

Fig. 4. Data save and sharing process

\subsection{Expert validation}

Before using the apps in the class and actual workplace, this apps has been presented to the ergonomics and education technology experts ( $\mathrm{N}=5$ persons), including the inventor for SERA form to evaluate the practicability of the apps for the field observation. Based on this evaluation, the SERA apps has been improved in term of its accessibility and its feature in digital form.

In the first design of SERA apps, the user as the evaluator was required to see the SERA manual form to view the pictures of the posture for each body part. After validating this procedure with the experts particularly experts from education technology, the SERA apps was redesigned to meet the expectation from them. Figure 5 shows an example of the improvement that has been done by the researcher. 


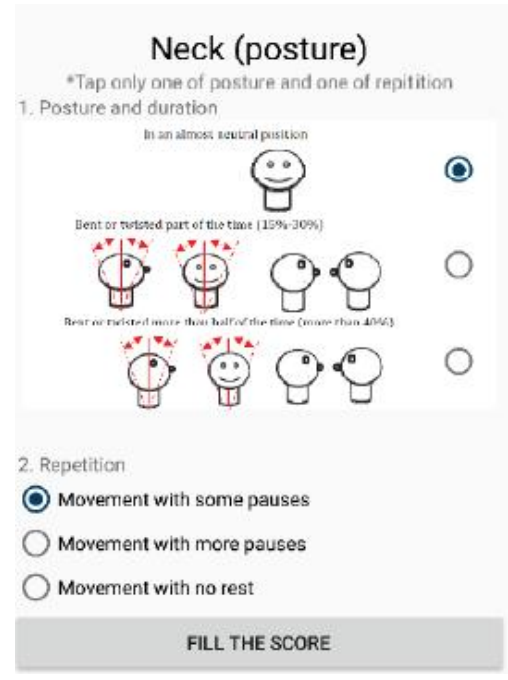

Fig. 5. Improvement on the current SERA apps based on the feedback from the expert

\subsection{Application of the SERA apps in the classroom}

After considering all feedbacks from the expert, this apps has been used by a group of manufacturing and mechanical engineering students (N=100 students) in the class of Quality Management and Manufacturing Ergonomics. From this session, the students were taught on how to use this apps to observe working condition in the workplace. In addition, the feedback from the students has been considered for further improvement. A readiness and perceptions on the apps usage has been gathered in this session based on the response from the students. In order to check the effectiveness of the SERA mobile apps, the design-based research (DBR) concept has been applied in this study. Based on Kinesh et al. [20], DBR is a method to bridge educational theory and practice by involving students' actual experiences and their feedback of those experiences. This concept was based on the idea from Kelly et al. and Middleton et al. [21-22]. Only students from Manufacturing Ergonomics class were selected to perform this task and the assessment was facilitated by a particular lecturer. There were about 20 fourth year undergraduate students involved in this study. They were selected because this risk assessment is one of the syllabus for this subject which is under design of manual handling scope. Therefore, their understanding on this field is better than those who did not enrolled in this class. Comparison between conventional method by using SERA manual form and the advanced method by using SERA mobile apps have been carried out in this study. Students need to provide their feedback based on the usage of both methods in group. The response was purely based on their experience when using manual form and mobile apps. 


\subsection{Application of the SERA apps in the actual environment as one of the practical learning}

As mentioned by past studies, the DBR concept should be applied not only in the formal setting [20-21 19]. In this case, formal setting is refers as a classroom environment. In order to use this concept, it also should be applied outside the classroom setting. Hence, the SERA apps has been used in the actual workplace by the practical engineering student for evaluating the condition of the work task. Recommendation and suggestion for work improvement can be given based on the final score from this risk assessment.

The risk assessment in the workplace was conducted at one of the car workshop in Selangor, Malaysia. The practical student provided the explanation regarding the benefit of the ERA method. In this study, the conventional assessment by using SERA manual form was shared with the owner and the employees in the workshop. Then, the digital technology by using SERA apps in tablet was compared with the manual form. These explanations were given to ensure the owner and employees understand the function and benefits of SERA for improving their working condition. In the meantime, based on this activity, it helps the student to improve communication and learning process by integrating the theory learned in the class and the actual condition when dealing with other parties outside the university. In this case, under Manufacturing Ergonomics subject, the student should understand and comprehend the basic concept of manual handling design and environment analysis. Hence, by observing the actual working condition, the student capable to relate the factors that help to minimize the risk of injury when performing manual handling task in the workplace. Environment improvement that relates with surrounding on the workplace also can be suggested based on the concept learned in the class. Figure 6 shows an activity that has been performed by the car maintenance worker. In this activity, the worker is required to fix a car motor starter. The load of the component is approximately $3.5 \mathrm{~kg}$.

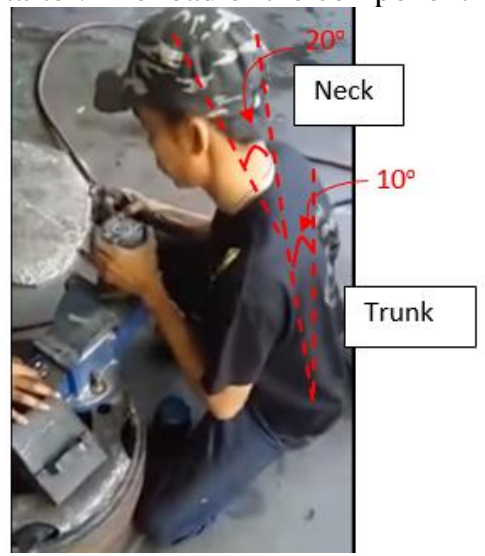

Fig. 6. Car maintenance worker 


\section{$3 \quad$ Result and Discussion}

This section provides the answer for three RQs as mentioned in Section 1. It includes the findings regarding effectiveness of the mobile apps application among the students in the university, particularly on those who were enrolled in the related subject. These findings provides the input for the first and part of second RQ. Then, findings from the actual assessment on the workplace condition was provided, which it helps to response to the third RQ and part of second RQ.

\subsection{Readiness assessment on the mobile apps}

Based on the response from the students, they provide positive feedback on the usage of digital form or in the mobile apps form for the SERA form. This is in-line with the past studies that mentioned in order to meet with the Net generation students, digital technologies are more appreciated by this group. It is highly valued as a new format which bot supports and improve the learning activities and can save time [19]. Hence, basically, the mobile apps capable to support the learning activities for the subject and in the classroom. Below are another main responses from the students:

- Majority of them mentioned mobile apps provides the benefit in term of fast calculation of the final result and can save the final results in the 'cloud' drive. Therefore, it is more 'green' compared to the manual form.

- Some of the students feel that it is more comfortable to see the contents of the apps by using the bigger size of mobile equipment. So, it is advisable to use tablet when using the SERA apps. It is due to there are a few figures inside this assessment that need to be seen by the user.

- The student feel happier to learn and use mobile applications as it suits with their generation.

\subsection{Findings of the risk assessment in the actual working environment by practical students}

According to SERA method, the worker was evaluated by using five factors including of posture, force, breaks, work pace, and other factor as mentioned in Section 3.0. As demonstrated in Figure 6, for this activity, the worker need to bent down his neck up to 200 and also his trunk up to 100 . Based on the theory from the subject in Manufacturing Ergonomics, posture is one the factors that contribute to risk of injury.

Based on the posture factor, the neck posture is bent or twisted more than half of the time. This is because of the component angle position cannot be adjustable, as illustrated in Figure 7. In term of neck repetition, there is a movement with some pauses. 


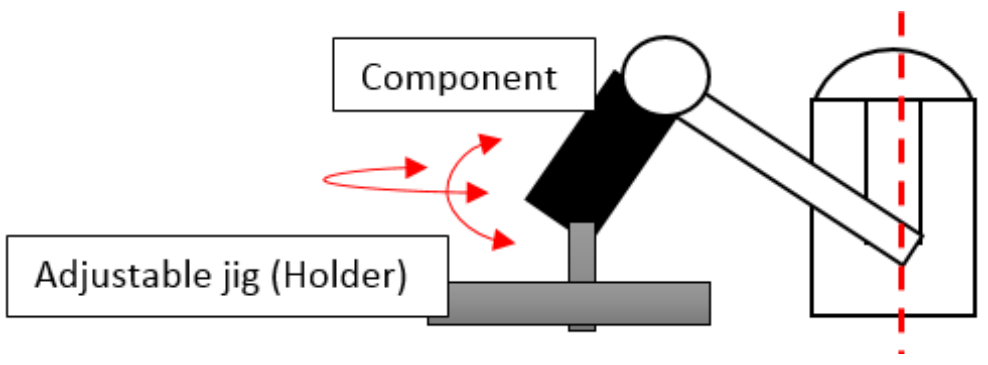

Fig. 7. Body posture with adjustable jig (component holder)

In term of trunk posture, the worker is in the bent forward position with more than half of the time and a lifting load is around 0 to $5 \mathrm{~kg}$. Then, lower arm and shoulder for the right and left body parts are in neutral position because both body parts are closed to the body. A wrist posture is bent more than half of the time with 0 to $5 \mathrm{~kg}$ load and over 20 times per minute of repetition for the both left and right of body parts. A hand posture is in pinch position with more than half of the time is in this position with 0 to $5 \mathrm{~kg}$ lifting load for the left and right body parts. A worker's leg posture is under squat position for both side. Table 1 summarized the final score for posture factor of each body part.

Table 1. Final score for posture factor

\begin{tabular}{|l|c|c|c|}
\hline \multicolumn{1}{|c|}{ Body part } & Initial score & Additional score & Final score \\
\hline Neck & 1 & 0 & 1 \\
\hline Trunk & 2 & 0 & 2 \\
\hline \multirow{2}{*}{ Shoulder } & Left: 0 & Left: 0 & Left: 0 \\
& Right: 0 & Right: 0 & Right: 0 \\
\hline \multirow{2}{*}{ Lower arm } & Left: 0 & Left: 0 & Left: 0 \\
& Right: 0 & Right: 0 & Left: 4 \\
\hline \multirow{2}{*}{ Wrist } & Left: 2 & Left: 2 & Right: 4 \\
\hline \multirow{2}{*}{ Hand } & Right: 2 & Right: 2 & Left: 4 \\
& Left: 4 & Left: 0 & Right: 4 \\
\hline \multirow{2}{*}{ Leg } & Right: 4 & Right: 0 & Left: 6 \\
& Left: 6 & Left: - & Right: 6 \\
\hline & Right: 6 & Right: - & Left: 17 \\
& & Total: & Right: 17 \\
\hline
\end{tabular}

The second factor is regarding an existing force during fixing process of the car component. Figure 8 shows the illustration of the task for this activity. Based on this task, the exerted force is moderate for both body side. The score for this factor is 2 . In term of break as the third factor in the SERA method, the worker has one to two hours of continuous break for this task. The score for this factor is 2 . The next observation is regarding the work pace. The worker feel sometimes difficult to keep up with the work because of the muscle fatigue while performing the activity. The score for this factor is 1 . Then, the fifth factor is regarding another present factors for this activity. There is two factors present in this observation session 
- Insufficient lighting (only 300 lux)

- The presence of vibration source from the equipment to open the bolt and nut. The score for this factor is 2 .

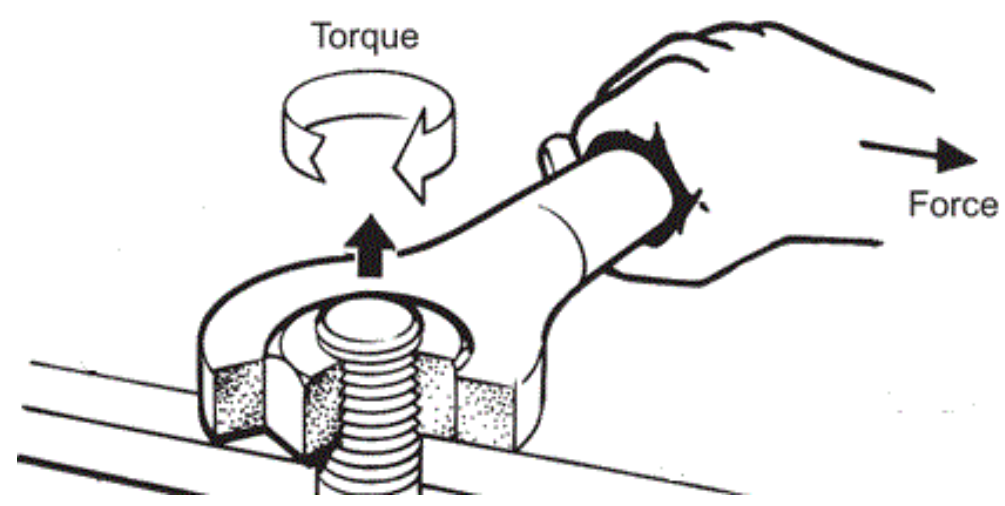

Fig. 8. Force exerted during opening the nut

The total score based on five factors in SERA method for this observation is 24 for both sides. The SERA result shows the medium ergonomic risk exposure level and therefore, suggestion for further investigation and changes is required. Based on the result, there are a few improvements can be considered such as provision of sufficient facilities (eg: lighting) for the workplace and also flexible as well as adjustable jig and fixture. Based on Manufacturing Ergonomics subject, under manual handling design and environment analysis, a few recommendations can be used to improve the working condition [16]. Overhaul or maintenance activity in the car workshop can be categorised as performance of visual tasks of medium contrast or small size. Hence, the illuminance range of recommendation is around 500 to 1000 lux [23]. Then, to prevent the awkward posture, the flexible and adjustable jig and fixture position can help to minimize the awkward posture during fixing the car component. All in all, this SERA apps helps to provide rapid result on the risk assessment at the workplace. The student can relate practical experience in the actual workplace with theories found in the university subject. It is in-line with the past studies that mentioned technology capable to make learning more accessible, efficient and experiences more memorable $[3,4]$. In addition, mobile apps can help to ease the empirical investigation and research [8].

The key finding for this research is that effective integrated learning aids enhanced learner engagement with valuable learning experiences. This mobile tool is extremely useful for various parties, due to an integrated information rich tool that is related to risk factors when performing a task, user friendly and interactive features and it can be used by various mediums; either by computer or telephone. 


\section{Conclusion}

This new application accommodates the needs of current student's and industry preferences; that prefer to study using an interactive approach and can be used during practical session. SERA was selected to be developed in the risk assessment app because this technique is recent and able to cover many factors to evaluate ERA. The app, which was developed using Android programming code, can be accessed via computer, laptop, tablet or mobile phones. Therefore, the new app is in both mobile and portable forms and has user friendly features. Some concluding observations from this new development for education purpose are as follows;

- Educational apps can support and enhance learning practises. Mobile apps can promote entertainment and provide active learning. It can increase the number of interactive learning activities and, simultaneously, improve quality of learning by making things easier for students to understand.

- Most importantly, educational apps are more environmentally friendly. This means a greener earth for future generations, because mobile educational apps only require downloading via an online system.

- The use of apps is highly valued by students; and new formats are beneficial to them and industry.

- For industry, it helps to improve the working condition of the worker in the company. As for example, in this paper, the practical student helps the company to determine the risk of working condition for this current activity. Based on this observation, there is moderate ergonomic risk exposure level to the worker. Hence, the current practice for this activity should be improved. Based on the observation, there are two recommendations for the company, in term of improvement on the facilities (eg: lighting) and equipment (eg: provide adjustable jig and fixture).

\section{Acknowledgement}

Part of this research was supported by FRGS/1/2018/TK03/UKM/03/2 and Universiti Kebangsaan Malaysia.

\section{References}

[1] Fauzi, S. N. F. M. (2013). Students' performance in practical training: Academicians evaluation. Procedia-Social and Behavioral Sciences, 93: 1275-1280. https://doi.org/10.1016/j. $\underline{\text { sbspro.2013.10.028 }}$

[2] Martin, L. K., \& Slater, C. (2012). Should Engineering Education be More Practical, More Theoretical, or Remain As it Currently Stands? [The Way I See It]. IEEE Potentials, 31(4): 6-8. https://doi.org/10.1109/mpot.2012.2195217

[3] Al Musawi, A. S. (2011). Redefining technology role in education. Creative Education, 2(02): 130. 
[4] Bahr, N. J. (2018). System safety engineering and risk assessment: a practical approach. CRC press.

[5] Khamis, N., Abrahman, M. N., Jamaludin, K. R., Ismail, A. R., Ghani, J. A., \& Zulkifli, R. (2009, July). Development of 5S practice checklist for manufacturing industry. Proceedings of the World Congress on Engineering, 1(3): 1-5.

[6] Persson, V., \& Nouri, J. (2018). A systematic review of second language learning with mobile technologies. International Journal of Emerging Technologies in Learning, 13(2). https://doi.org/10.3991/ijet.v13i02.8094

[7] Vázquez-Cano, E. (2014). Mobile distance learning with smartphones and apps in higher education. Educational Sciences: Theory and Practice, 14(4): 1505-1520. https://doi.org/ 10.12738/estp.2014.4.2012

[8] Williams, A. J., \& Pence, H. E. (2011). Smart phones, a powerful tool in the chemistry classroom. Journal of Chemical Education, 88(6): 683-686. https://doi.org/10.1021/ed2000 $29 \mathrm{p}$

[9] Md Jamil, M.S, Abdul Wahab, N.A., Rajikan, R. Saat, M.H.,Sannusi, S.N., (2019). Kebolehgunaan Dan Kesan Aplikasi Mudah Alih (Mobile Apps) Dalam Minggu Mesra Mahasiswa, Jurnal Personalia Pelajar, 93-101.

[10] Zainun, R., Lam, M.C., Bakar, K.A.A. and Ismail, A.K., 2018. User Experience Model for Remote Envenomation Consultation Mobile Application with Decision Support Ability. International Journal on Advanced Science, Engineering and Information Technology, 8(42):.1470-1479. https://doi.org/10.18517/ijaseit.8.4-2.6836

[11] Arshad, H., Obeidy, W.K. and Abidin, R.Z., 2017. An interactive application for halal products identification based on augmented reality. International Journal on Advanced Science, Engineering and Information Technology, 7(1):.139-145. https://doi.org/10.18517/ija seit.7.1.1793

[12] Chen, Y., Wong, J., Ayob, A., Othman, N. and Poh, B., 2017. Can Malaysian young adults report dietary intake using a food diary mobile application? A pilot study on acceptability and compliance. Nutrients, 9(1), p.62. https://doi.org/10.3390/nu9010062

[13] Eppard, J., Nasser, O., \& Reddy, P. (2016). The Next Generation of Technology: Mobile Apps in the English Language Classroom. International Journal of Emerging Technologies in Learning, 11(4). https://doi.org/10.3991/ijet.v11i04.5293

[14] Occupational Safety and Health Administration. "Ergonomics: The study of work," 16 November 2017; www. osha. Gov.

[15] Otto, A., \& Battaïa, O. (2017). Reducing physical ergonomic risks at assembly lines by line balancing and job rotation: A survey. Computers \& Industrial Engineering, 111: 467480. https://doi.org/10.1016/j.cie.2017.04.011

[16] Sukadarin, E. H., Deros, B. M., Ghani, J. A., Ismail, A. R., Nawi, N. S. M., \& Abdull, N. (2016). Validity test for Simple Ergonomics Risk Assessment (SERA) method. Malaysian Journal of Public Health Medicine, 1(Specialissue1): 134-143.

[17] Rahamat, R.B., Shah, P.M., Din, R.B. and Aziz, J.B.A., 2017. Students'readiness and Perceptions towards Using Mobile Technologies For Learning The English Language Literature Component. The English Teacher, p.16.

[18] Jan, S.R., Ullah, F., Ali, H. and Khan, F., 2016. Enhanced and effective learning through mobile learning an insight into student's perception of mobile learning at University Level. International Journal of Scientific Research in Science, Engineering and Technology (IJSRSET), pp. 2395-1990.

[19] Vázquez-Cano, E., 2014. Mobile distance learning with smartphones and apps in higher education. Educational Sciences: Theory and Practice, 14(4), pp.1505-1520. https://doi. org/10.12738/estp.2014.4.2012 
[20] Kinash, S., Brand, J. and Mathew, T., 2012. Challenging mobile learning discourse through research: Student perceptions of Blackboard Mobile Learn and iPads. Australasian journal of educational technology, 28(4). https://doi.org/10.14742/ajet.832

[21] Middleton, J., Gorard, S., Taylor, C. and Bannan-Ritland, B., 2014. 2 The "Compleat” Design Experiment1. Handbook of Design Research Methods in Education: Innovations in Science, Technology, Engineering, and Mathematics Learning and Teaching, p.21.

[22] Kelly, A.E., Lesh, R.A. and Baek, J.Y. eds., 2014. Handbook of design research methods in education: Innovations in science, technology, engineering, and mathematics learning and teaching. Routledge.

[23] Mital, A., Kilbom, Å., \& Kumar, S. (Eds.). (2000). Ergonomics guidelines and problem solving (Vol. 1). Elsevier. https://doi.org/10.1016/s1572-347x(00)80001-4

\section{$7 \quad$ Authors}

Nor Kamaliana Khamis and Amirul Mukhlish Abdul Azam are from Faculty of Engineering \& Built Environment in Universiti Kebangsaan Malaysia in Bangi of Selangor in Malaysia.

Article submitted 2019-09-23. Resubmitted 2019-10-27. Final acceptance 2019-11-05. Final version published as submitted by the authors. 